\title{
Feasibility of enhanced recovery after surgery program in colorectal surgery during COVID-19 pandemic in Italy: should we change something?
}

\author{
Felice Borghi ${ }^{1}$ (D . Luca Pellegrino ${ }^{1} \cdot$ Vincenzo Pruiti $^{1} \cdot$ Danilo Donati $^{1} \cdot$ Giorgio Giraudo ${ }^{1}$
}

Received: 22 May 2020 / Accepted: 31 May 2020 / Published online: 13 June 2020

(C) Italian Society of Surgery (SIC) 2020

Coronavirus disease 2019 (COVID-19) spreading has created unprecedented challenges for healthcare systems. Nonurgent and non-cancerous procedures have been cancelled to permit reallocation of nurses and anesthesiologists, operating theatres were partially converted into intensive care unit beds and, consequently, a delay in the management of oncological patients was recorded. International recommendations propose pre-hospitalization swab testing, restriction of visitors, physical distance, adoption of measures to decrease hospital stay, postponement elective surgical and endoscopic cases, and evaluation of correct indications for minimally invasive approach.[1].

In our tertiary care hospital, the total number of 700 beds was reduced and 150 were reserved for COVID positivity with a dedicated inpatient pathway. Our surgical ward was a COVID-free service and one ICU was maintained for patients with negative swab.

In recent years enhanced recovery after surgery program (ERAS), mostly in colorectal surgery, has shown interesting results in terms of reduction in post-operative complications, hospital stays and costs. At our centre, ERAS pathway was implemented in 2010 and, so far, over 1300 patients have been included in the protocol $[2,3]$. During the lockdown period (March-April 2020), adopting specific guideline recommendations for preserving COVID-19 transmission, we continued to apply the same program, treating 24 patients (16 males and 8 women) with mean age of 68.2 years. All patients had a preoperative swab test COVID negative. The indication for surgery was colorectal malignancy $(91.7 \%)$, or severe diverticular disease (8.3\%). Anterior rectal resection was performed in 10 patients $(41.7 \%)$, while colonic resection in 14 patients (58.3\%). Protective ileostomy was carried

Felice Borghi

borghi.f@ospedale.cuneo.it

1 ASO Santa Croce e Carle, Cuneo, Italy out in seven cases (30.4\%). Sixteen patients $(66.7 \%)$ were treated with a traditional laparoscopic technique and seven (29.2\%) using the DaVinci Xi robotic system. Minimally invasive surgical procedures were performed in according to international recommendations (personal protective equipment, $\mathrm{CO} 2$ filter, negative pressure room, smoke evacuation system). The median post-operative stay was 4 days (range 2-12 days), both for colonic and rectal surgery. One patient (4.2\%) developed intra-abdominal collection, drained under TC-scan guide. Minor complications occurred in 10 patients $(41.7 \%)$ and $8.3 \%$ were readmitted. No patient has developed post-operative respiratory complications and no patient required an ICU stay. The mean adherence to the ERAS pathway was $82.6 \%$. Adhesion to preadmission items was $76.1 \%$; for pre, intra and post-operative elements, the mean compliance was $90 \%, 72.9 \%$, and $85.6 \%$, respectively. The less adherence was recorded for counselling $(41.7 \%)$, scheduled mobilization $(45.8 \%)$ and intra-operative restrictive fluid administration (58.3\%) (Fig. 1). A decrease of overall compliance must be considered for the need to reduce hospital access and interpersonal contacts and some modifications of the traditional ERAS program need to be reorganized. In preadmission phase, we suggest a limited 1 day hospital access to perform blood test, oncological markers, CT scan, nursing evaluation, nutritional screening, psychological and fragility assessment. Multidisciplinary tumour board should be performed using teleconference. A personalized kit with specific program brochure, carbohydrate drinks, preoperative venous thromboembolism prophylaxis and oral iron supplementation when necessary should be administered. Hospitalization should be planned always the same day of surgery. The predictable reduced compliance to the scheduled mobilization, due to the reduced access to common areas and to the limitations of hospital visits for caregivers and physiotherapists, could be exceeded using patients' onsite mobilization, such as, treadmill, exercise bike, or steps. 
Fig. 1 Adhesion to ERAS items

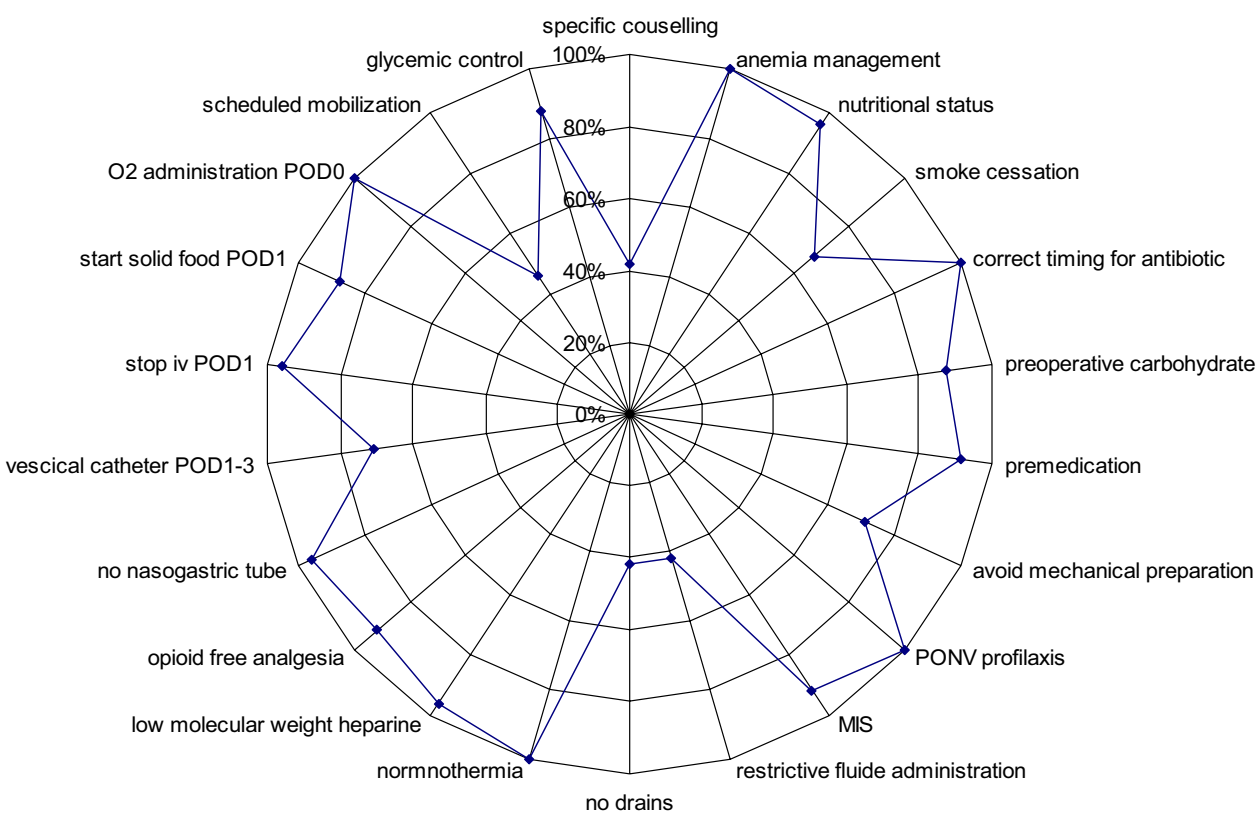

Post-operative follow-up, performed using telemedicine in some cases, could be improved in the future.

The implementation of ERAS pathway is complex and cannot be carried out in a period of health crisis. The centralization of cases towards ERAS experienced centres, during pandemic, perhaps, could help to optimize peri-operative results. The aim of healthcare system should be treating as many patients as possible, trying to optimize post-operative outcomes and to respect the timing between cancer diagnosis and surgical treatment. We believe that the ERAS pathways retain an important role even in a period of organizational difficulties such as the COVID-19 pandemic. More studies are needed to confirm these preliminary data.

Author contributions All authors have contributed to the work and agreed on the final version.

Funding None.

\section{Compliance with ethical standards}

Conflict of interest The authors declare that they have no conflict of interest.

Research involving human participant and/or animals All procedures performed in studies involving human participants were in accordance with the ethical standards of the institutional and/or national research committee (include name of committee + reference number) and with the 1964 Helsinki declaration and its later amendments or comparable ethical standards.
Informed consent Informed consent was obtained from all individual participants included in the study.

\section{Reference}

1. Coccolini F, Perrone G, Chiarugi M, Di Marzo F, Ansaloni L, Scandroglio I, Marini P, Zago M, De Paolis P, Forfori F, Agresta F, Puzziello A, D’Ugo D, Bignami E, Bellini V, Vitali P, Petrini F, Pifferi B, Corradi F, Tarasconi A, Pattonieri V, Bonati E, Tritapepe L, Agnoletti V, Corbella D, Sartelli M, Catena F (2020) Surgery in COVID-19 patients: operational directives. World J Emerg Surg 15(1):25. https://doi.org/10.1186/s13017-020-00307 $-2$

2. Gustafsson UO, Scott MJ, Hubner M, Nygren J, Demartines N, Francis N, Rockall TA, Young-Fadok TM, Hill AG, Soop M, de Boer HD, Urman RD, Chang GJ, Fichera A, Kessler H, Grass F, Whang EE, Fawcett WJ, Carli F, Lobo DN, Rollins KE, Balfour A, Baldini G, Riedel B, Ljungqvist O (2019) Guidelines for perioperative care in elective colorectal surgery: enhanced recovery after surgery $\left(\right.$ ERAS $^{\circledR}$ ) society recommendations: 2018. World J Surg 43(3):659-695. https://doi.org/10.1007/s00268-018-4844-y

3. Braga M, Borghi F, Scatizzi M, Missana G, Guicciardi MA, Bona S, Ficari F, Maspero M, Pecorelli N, PeriOperative Italian Society (2017) Pecorelli N (2017) Impact of laparoscopy on adherence to an enhanced recovery pathway and readiness for discharge in elective colorectal surgery: results from the PeriOperative Italian Society registry. Surg Endosc. 31(11):4393-4399. https://doi. org/10.1007/s00464-017-5486-0

Publisher's Note Springer Nature remains neutral with regard to jurisdictional claims in published maps and institutional affiliations. 